\title{
Glazialhydrologisches Monitoring im Bereich des Rauriser Sonnblicks (Hohe Tauern, Österreich)
}

\author{
Glaciohydrological monitoring in the region of Rauriser Sonnblick (Hohe Tauern, Austria)
}

von W. Schöner ${ }^{1}$, B. Hynek¹, R. Böhm¹, D. Binder², G. Koboltschnig ${ }^{3}$, H. Holzmann ${ }^{3}$

\section{Kurzfassung/Summary}

In diesem Beitrag wird das glazialhydrologische Monitoring im Bereich der Rauriser Arche (Rauriser Sonnblick) beschrieben. Das Monitoring zeichnet sich durch langjährige und umfangreiche Messungen der Gletschermassenbilanz, des Glazialabflusses und der räumlich verteilten Niederschlagmenge und der Schneehöhe aus. Alle untersuchten Gletscher sind kleiner als $1.5 \mathrm{~km}^{2}$. Im Gegensatz zu dem ebenfalls sehr detaillierten Gletschermonitoring im Bereich der Rofenarche (Tirol), befindet sich das Sonnblickgebiet in einer sehr niederschlagsreichen Region. Dieser markante Unterschied in der Niederschlagsmenge hat eine deutlich höhere Klimasensitivität der Gletscher im Sonnblickgebiet zur Folge. Ein Vergleich der vorliegenden Ergebnisse für die 3 Gletscher des Monitorings im Bereich des Sonnblicks zeigt die große Bedeutung des Alpenhauptkammes als Klimascheide. So weist das nördlich gelegene Goldbergkees deutlich höhere Winterakkumulationen auf als die südlich gelegenen Gletscher Wurtenkees und Kleinfleißkees. Ergebnisse von kürzlich durchgeführten Messungen der Eismächtigkeiten zeigen für die untersuchten Gletscher maximale Eismächtigkeiten zwischen ca. 80 bis $150 \mathrm{~m}$. Die vorliegenden Daten des Monitorings eignen sich sehr gut für die Kalibrierung and Validierung glazialhydrologische Modelle.

The paper describes the glaciohydrological monitoring in the region of Rauriser Sonnblick, which is charcterised by long-term, comprehensive measurements of the glacier

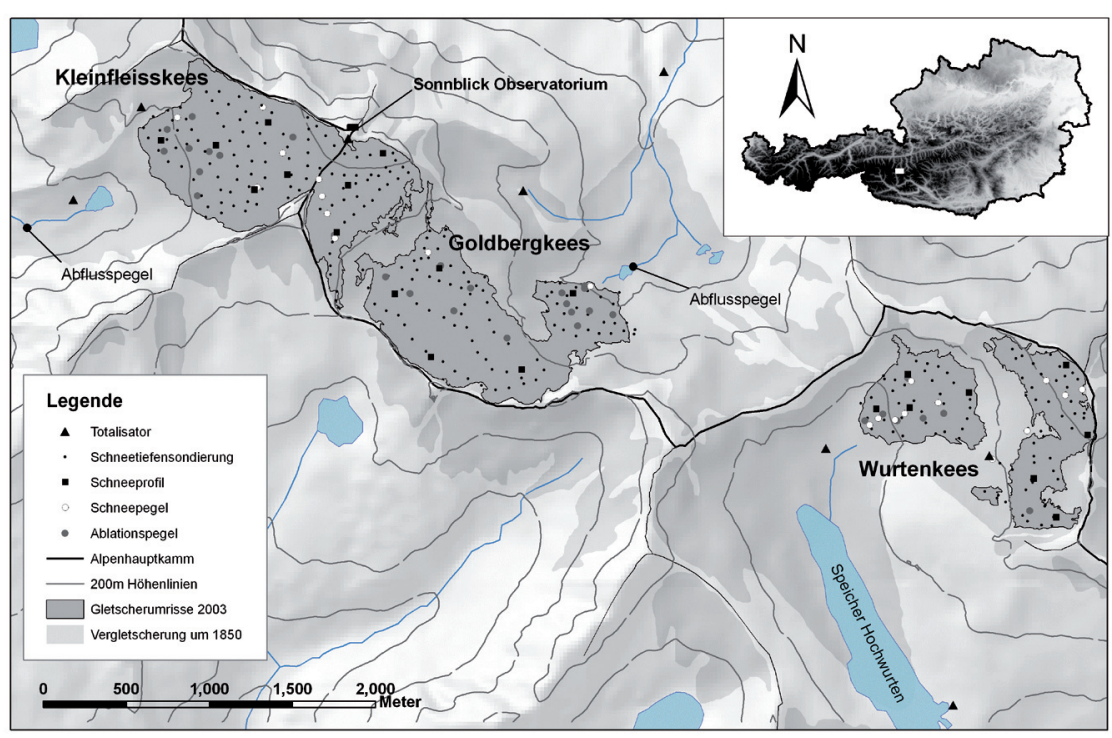

Abb. 1: Messnetz des Gletschermonitorings im Bereich des Rauriser Sonnblicks

mass balance, the glacial discharge and the spatial distribution of precipitation and snow height. All investigated glaciers are less than $1.5 \mathrm{~km}^{2}$ in size. Annual precipitation in the Sonnblick region is high compared to another well established glacier monitoring in Austria (Rofenarche region, Tyrol). Thus climate sensitivity of glaciers in the Sonnblick region is much higher compared to the Rofenarche area. The analysis of available monitoring data from Sonnblick region reveals the high significance of the Alpine crest as a distinct climate divide. Consequently, winter accumulation is much more pronounced at Goldbergkees (north of the Alpine crest) compared to the Wurtenkees or Fleißkees which are situated south of the Alpine crest. Results from recently performed ice thickness measurements show values between approximate 80 to $150 \mathrm{~m}$ maximum ice thickness. The today status of the glacier monitoring in the Sonnblickregion is especially valuable for the calibration and validation of glaciohydrological models.

\section{Einleitung}

Die Bedeutung der Gletscher für die Klimatologie und Hydrologie ist durch eine Vielzahl von Studien belegt und zeigt sich auch deutlich in der umfangreichen Behandlung im 4ten Bericht des IPCC (2007). Für die Untersuchung des Zusammenhanges zwischen dem Gletscherverhalten und dem Klima beziehungsweise der Abflussbildung ist die Erfassung der Gletscheränderungen notwendig. Strategien für derartige Beobachtungssysteme der Gletscher (Monitoring) wurden vielfach dargelegt und sind durch die Aktivitäten des World Glacier Monitoring Service (Haeberli et al., 2007) oder des World Data Center for Glaciology auf internationaler Ebene verankert (Dyurgerov and Meier, 2005). Die Anzahl der gemessenen Gletscher ist jedoch im Vergleich zur Gesamtzahl der 
Gletscher sehr klein und beschränkt sich auf logistisch gut erreichbare und leicht $\mathrm{zu}$ messende Gletscher. Es ist daher klar, dass die Stichprobe der gemessenen Gletscher die Grundgesamtheit aller Gletscher nur schlecht wiedergibt (Zemp, 2006). Außerdem wird an vielen Gletschern nur die Nettomassenbilanz über das gesamte Jahr gemessen und nicht die Wintermassenbilanz und die Sommermassenbilanz getrennt erfasst. Monitoringprogramme die über die Messung der Massenbilanz hinausgehen und zusätzlich noch hydrologisch relevante Größen (Abfluss, räumliche Verteilung von Niederschlag und Schneehöhe) erfassen sind dann schon äußerst selten zu finden. Wurde ein glazialhydrologisches Monitoring einmal eingerichtet dann ist es von großer Wichtigkeit, dass es langfristig aufrecht erhalten werden kann. Nur wenn die Messserien lang genug sind können entsprechend abgesicherte Zusammenhänge zwischen dem Gletscherverhalten und dem Klima sowie der zeitlichen Änderung des glazialen Abflusses erkannt werden. $\mathrm{Zu}$ den herausragenden langjährigen Monitoringprogrammen in Europa zählen derStorglaciären in Schweden(Homlund and Jansson, 2005) oder der Vernagtferner im Ötztal (Escher-Vetter et al., 2005). Das Monitoringprogramm im Bereich des Rauriser Sonnblicks bekommt auf Grund seiner Lage in unmittelbarer Nähe zum Sonnblick Observatorium, mit seinem umfassenden Messprogramm, zunehmend einen besonderen Stellenwert. Die wesentlichen Grundlagen, die zeitliche Entwicklung sowie einige wesentlichen Erkenntnisse dieses Monitorings sollen im Folgenden genauer beschrieben werden.

\section{Das Glazilahydrologische Monitoring im Sonnblick- gebiet}

Das Sonnblick Observatorium hat durch seine Lage in unmittelbarer Nähe zu den größten Gletschern

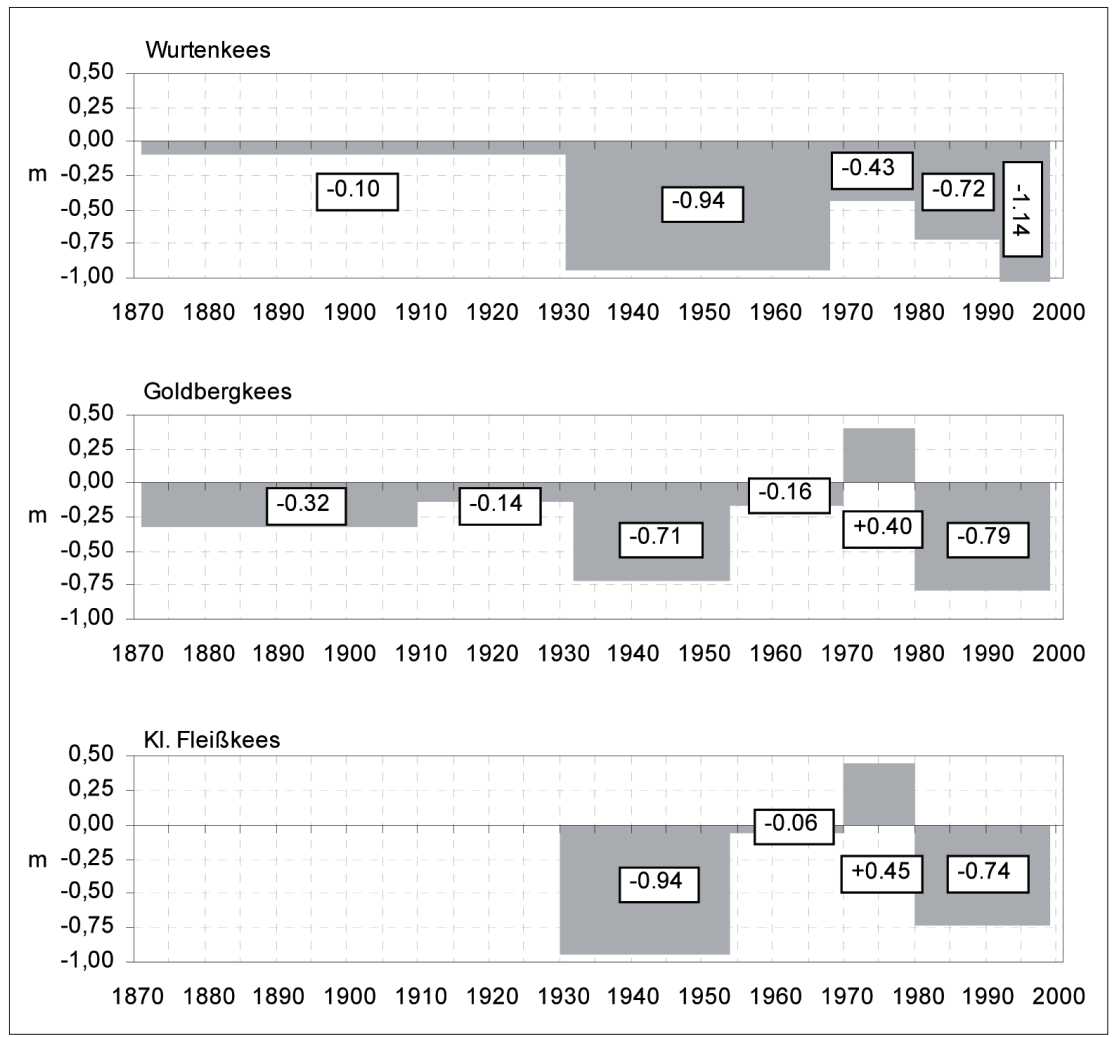

Abb. 2: Mittlere Höhenänderungen pro Jahr der 3 Hauptgletscher (Wurtenkees, Goldbergkees und Kleinfleißkees) im Sonnblickgebiet seit den ersten Kartenaufnahmen

Tab. 1: Charakteristische Maßzahlen der Gletscher im Bereich des

Rauriser Sonnblicks sowie Jahreszahlen des Beginns der verschiedenen Teilbereiche des Gletschermonitorings

\begin{tabular}{lccc} 
& Wurtenkees & Goldbergkees & Kleinfleißkees \\
\hline Fläche (2003) & 0,9 & 1,8 & 1 \\
\hline $\begin{array}{l}\text { Exposition Akkumulations- } \\
\text { gebiet (2003) }\end{array}$ & SW & SE & W \\
\hline $\begin{array}{l}\text { Exposition Ablationsgebiet } \\
\text { (2003) }\end{array}$ & SW & NE & W \\
\hline Fotodokumentation & 1896 & 1829 & 1926 \\
\hline Klimastation & 1886 & 1886 & 1886 \\
\hline Längenmessung & 1896 & 1896 & 1896 \\
\hline Schneehöhenmessungen & 1982 & 1928 & 1928 \\
\hline $\begin{array}{l}\text { Niederschlagsmessung } \\
\text { (Totalisatoren) }\end{array}$ & 1982 & 1928 & 1928 \\
\hline Winterbilanzmessung & 1983 & 1987 & 1999 \\
\hline Nettomassenbilanz & 1983 & 1989 & 1999 \\
\hline Abflussmessung & & 2002 & 2004 \\
\hline Schneechemie & 1983 & 1987 & \\
\hline
\end{tabular}

der Goldberggruppe seit Beginn das Interesse der Glaziologie auf sich gezogen. Erste Meilensteine glaziologischer Arbeiten waren die früher kartographischen Aufnahmen (erste terrestrische Aufnahme eines Gletschers im Jahr 1909, siehe Hübl, 1912) sowie der Beginn der Längenänderungsmessungen im Jahr 1896 durch Albrecht Penck(Penck, 1897). 
Bereits 1927 wurde ein Messnetz zur Erfassung der Schneehöhe und des Niederschlages für das Gebiet von Kleinfleißkees und Goldbergkees eingerichtet, das wichtige Informationen über Akkumulation und Ablation auf dem Gletscher liefern kann (siehe Schöner et al., 2009). Pionierarbeit für ein glaziologisches Monitoring leistete Norbert Lichtenecker in den 1930er Jahren, der bereits Überlegungen zur Messung der Massenbilanz des Goldbergkees anstellte (Lichtenecker 1936a, 1936b, 1938). Leider wurden diese Aktivitäten durch den 2. Weltkrieg und den Tod Norbert Lichteneckers abrupt abgebrochen. Weitere herausragende glaziologische Untersuchungen waren die Arbeiten von Franz Sauberer und Inge Dirmhirn die umfangreiche Messungen über den Strahlungshaushalt über Gletscherflächen durchführten (Dirmhirn und Sauberer, 1952, Sauberer und Dirmhirn, 1950) und die Bedeutung der Albedo für die sommerliche Abschmelzung erkannten (Sauberer, 1938, Dirmhirn 1961). Mehrere Studien über das Gletscherverhalten wurden auch von Tollner durchgeführt (z. B. Tollner, 1978), der auch über lange Zeit die Längemessungen ausführte.

Der Anstoß für das Monitoring in seiner heutigen Form erfolgte durch die Aufnahme der Massenbilanzmessungen am Wurtenkees im Jahr 1982 durch R. Böhm und N. Hammer. Dabei wurde erstmals in Österreich nicht nur die Jahresmassenbilanz, sondern auch die Wintermassenbilanz für einen Gletscher getrennt gemessen. Es ist ein besonderes Charakteristikum der Massenbilanzmessungen im Sonnblickgebiet, dass der Beginn der Messreihe auf die markante Änderung der Massenbilanzen von eher positiven auf deutlich negative Haushaltsjahre fällt. Die Massenbilanzmessungen am Wurtenkees wurden 1987 auf das Goldbergkees und 1999 auf das Kleinfleißkees ausgeweitet. Motivation für die Ausdehnung der Messungen war die Erfassung der räumlichen Variabilität des Gletscherverhaltens über den Alpenhauptkamm als markante Klimascheide. Diese markante Klimascheide zeigt sich besonders in der Karte der räumlichen Verteilung des Niederschlags (Auer et al., 2002)

Neben dem umfangreichen glaziologischen Monitoring im Bereich der Rofener Arche (Hintereisferner, Kesselwandferner, Vernagtferner), ist das Gletschermonitoring im Bereich des Rauriser Sonnblicks das umfangreichste in den österreichischen Alpen. Vergleicht man die zwei Monitoringgebiete, dann fällt der deutliche Unterschied in der Niederschlagssumme auf (Jahresniederschlagssumme im Bereich von Hintereisferner und Vernagtferner ca. $1500 \mathrm{~mm}$, im Bereich des Goldbergkees ca. $2500 \mathrm{~mm}$ ). Eine Auflistung aller Gletschermonitoringprogramme in Österreich findet sich in Gattermayr et al. (2005).

Die Massenbilanz der 3 Hauptgletscher im Sonnblickgebiet wird seit Beginn für jedes Einzeljahr mit der glaziologischen Methode gemessen (Hoinkes 1970, Kaser et al., 2003). Zusätzlich erfolgt auch eine Messung der Volumsunterschiede beziehungsweise mittleren Höhenänderungen mittels der sogenannten geodätischen Methode (Finsterwalder, 1953). Die Daten über Höhenänderungen gehen für die Goldberggruppe bis zum Jahr 1871 zurück (Abb. 2). Diese Daten belegen sehr gut den markanten Gletscherrückgang seit dem Ende der Kleinen Eiszeit um 1850.

Neben der Massenbilanz und den Volums- beziehungsweise Höhenänderungen der 3 Gletscher wird das Gletschermonitoring durch eine Vielzahl anderer Messungen und Beobachtungen ergänzt (Tab. 1). Herausragend ist dabei das schon sehr lange zurückreichende Messnetz der räumlichen Verteilung des Niederschlags (mit monatlich abgelesenen Totalisatoren) und das Messnetz zur Erfassung der räumlichen Verteilung der Schneehöhen. Ein wesentlicher Schritt zur Vervollständigung des Monitorings wurde 2002 durch die Messung des Abflusses gesetzt. 2004 wurden diese Messungen, in Zusammenarbeit mit der KELAG, auf das Kleinfleißkees ausgedehnt.

\section{Die Massenbilanz der Glet- scher im Sonnblickgebiet}

Die drei Gletscher Wurtenkees, Goldbergkees und Kleinfleißkees liegen räumlich unmittelbar nebeneinander, wobei das Goldbergkees nördlich des Alpenhauptkammes, das Wurtenkees und das Kleinfleißkees südlich davon gelegen sind. Diese entfernungsmäßig geringen Unterschiede bewirken jedoch einen markanten topographischen Unterschied der sich auch entsprechend im Lokalklima äußert.

Die bisher vorliegenden Ergebnisse der Massenbilanzmessungen der 3 Gletscher sind in Abbildung 4 dargestellt. Während prinzipiell eine gute Übereinstimmung der Einzelreihen festgestellt werden kann, zeigt eine genauere Betrachtung auch signifikante Unterschiede zwischen den Zeitreihen. Folgende Schlussfolgerungen können aus dem Vergleich abgeleitet werden:

- Die Winterakkumulationen auf dem Goldbergkees sind im langjährigen Mittel markant höher als auf Wurtenkees und Kleinfleißkees.

- Die Winterakkumulationen auf Wurtenkees und Kleinfleißkees sind hoch miteinander korreliert und nahezu identisch vom langjährigen Mittel.

- Die Winterakkumulationen auf dem Wurtenkees zeigen einen leicht ansteigenden Trend, während für das Goldbergkees kein zeitlicher Trend festzustellen ist.

- Die Jahresbilanz des Goldbergkees ist im Mittel positiver als auf dem Wurtenkees. 


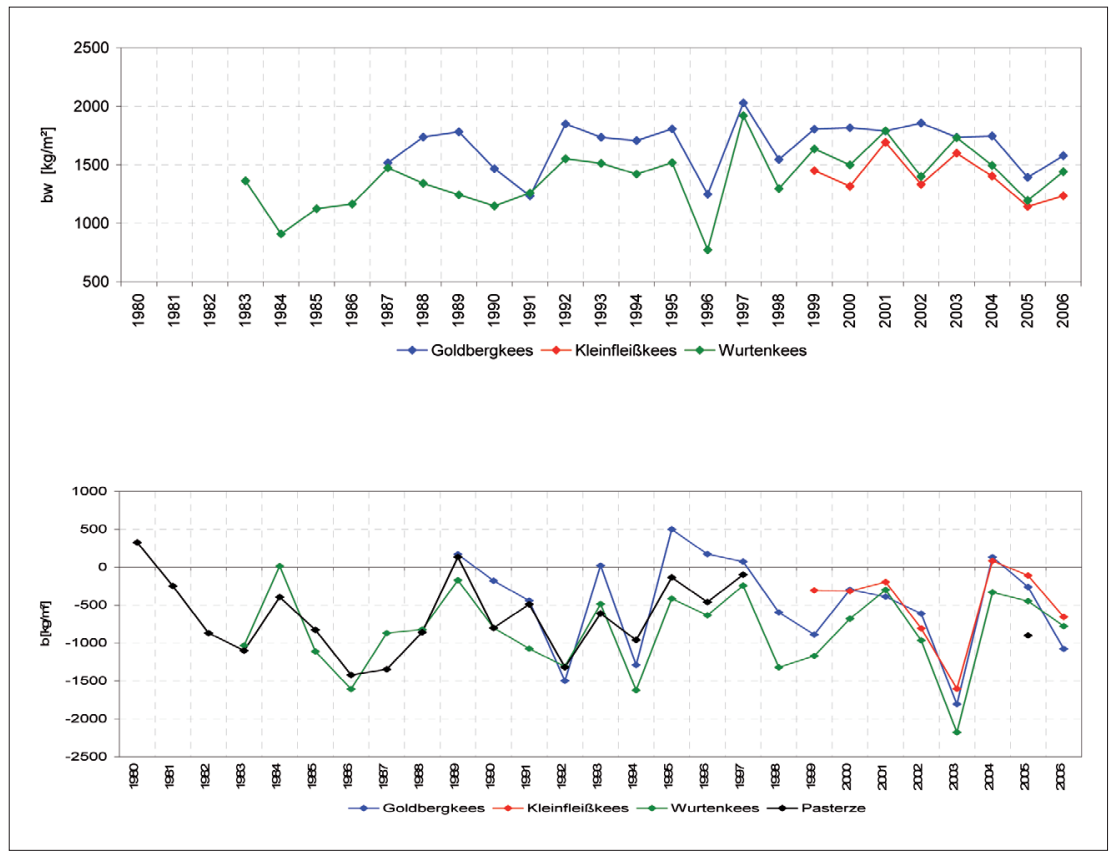

Abb. 3: Messreihen der Wintermassenbilanz bw (oben) und Nettojahresmassenbilanz b (unten) von Wurtenkees, Goldbergkees, Kleinfleißkees und Pasterze

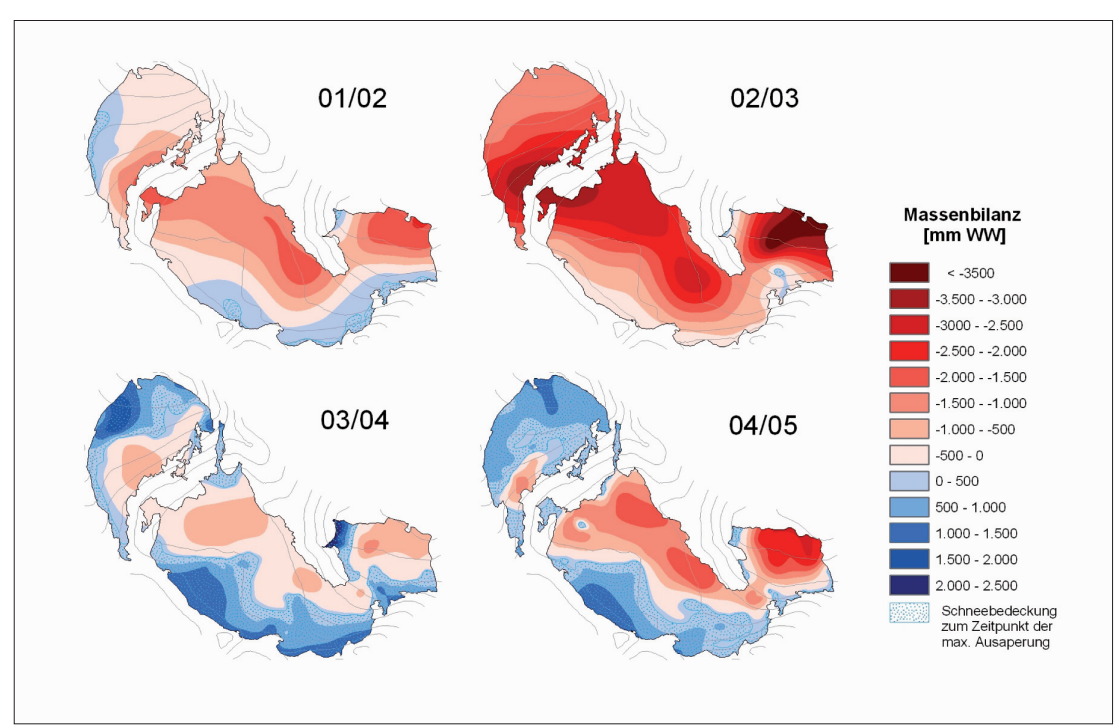

Abb. 4: Beispiele der räumlichen Verteilung der Massenbilanz auf dem Goldbergkees für verschiedene Haushaltsjahre (2002/2003 war ein extrem negatives Haushaltsjahr)

- Für die Jahresbilanzen von Wurtenkees und Goldbergkees ist ein schwach fallender Trend festzustellen.

- Auffällige Extremwerte der Massenbilanz waren für das Winterhalbjahr 1997 und für den Sommer 2003.

Die Massenbilanz der Gletscher im Sonnblickgebiet ist durch einen starken Einfluss der Orographie charakterisiert. Dies zeigt sich sowohl für die Wintermassenbilanz als auch für die Sommermassenbilanz (Abb. 5). In Folge dieses Einflusses besitzen die Gletscher mehrere orographisch bedingte Akkumulationsgebiete gewichtslinien in unterschiedlichen Seehöhen.

Neben der Massenbilanz ist auch und dadurch auch mehrere Gleich- die Eisbewegung (horizontal und vertikal) eine charakteristische Maßzahl über die Aktivität eines Gletschers. Derartige Messungen liegen von Kleifleißkees und Goldbergkees für das Jahr 2005/2006 vor (Tab. 2). Die Messungen zeigen überraschend hohe Werte der horizontalen Bewegungskomponente für das Kleinfleißkees (bis nahe $6 \mathrm{~m}$ pro Jahr). Diese hohen Werte sind jedoch recht gut verständlich, wenn man bedenkt, dass das Kleinfleißkees im Jahr 2002 die Verbindung zum Zungenende im unteren Steilbereich durch einen Gletschersturz verloren hat. In dem Bereich oberhalb dieser Abrissstelle werden auch die hohen Bewegungswerte gemessen. Das Goldbergkees zeigt dagegen (obwohl flächenmäßig größer) Bewegungswerte die über $2.5 \mathrm{~m} / \mathrm{Jahr}$ nicht hinausgehen. Die Werte für die Vertikalbewegung der Gletscher sind alle kleiner als $1 \mathrm{~m}$ wobei sich die Richtungen der Vektoren, mit abwärts im Akkumulationsgebiet und aufwärts im Ablationsgebiet, gut mit den Erwartungen decken. Einzig der Pegel G04/5 zeigt eine Abwärtsbewegung im Ablationsgebiet. Diese ist aus dem starken Einsinken auf Grund der starken Hohlraumbildung in diesem Bereich zu verstehen.

\section{Eismächtigkeit}

Neben der Erfassung der Massenänderungen eines Gletschers ist auch die Bestimmung der Eismächtigkeit und des Eisvolumens eine wesentliche Datengrundlage für ein Gletschermonitoring. Die Mächtigkeit der Gletscher der Goldberggruppe wurde in den 1960er/1970er Jahres mittels Refraktionsseismik gemessen (Brückl, 1977). Im Zuge der Massenbilanzmessungen zeigte sich jedoch recht bald, dass diese Messungen die Eismächtigkeiten nicht richtig erfasst hatten. Im Rahmen eines Projektes und zweier Diplomarbeit wurden die Eismächtigkeit mittels Ground Pentrating Radar (GPR) 2003 und 2004 neu vermes- 


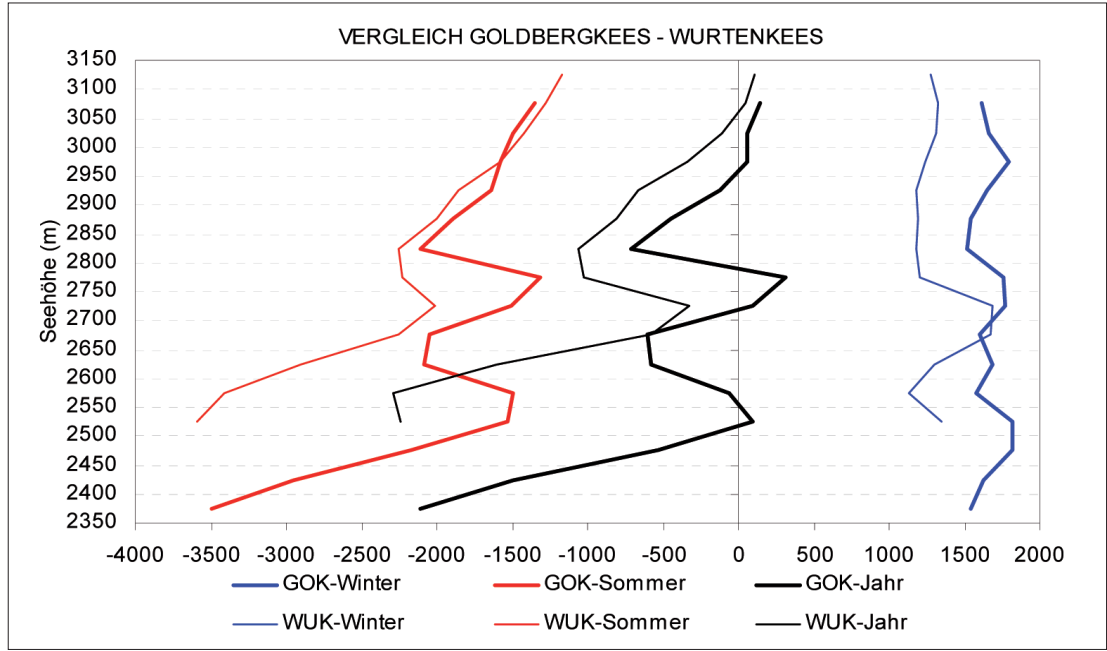

Abb. 5: Höhenabhängigkeit der spezifischen Massenbilanz (getrennt nach Winter, Sommer und Jahr) von Goldbergkees (GOK) und Wurtenkees (WUK). Dargestellt sind gemittelte Kurven über die Periode 1987 bis 2002.

sen (siehe Binder, 2008, Binder et al., 2009). Als wesentliches Ergebnis dieser Messungen ist festzuhalten, dass die doch sehr kleinen Gletscher im Bereich des Rauriser Sonnblicks noch Maximaltiefen größer als $140 \mathrm{~m}$ erreichen.

\section{Zusätzliches Schnee- monitoring während des Projektes SNOWTRANS}

Im Rahmen des Projektes Snowtrans wurde das Monitoring im Bereich des Goldbergkees etwas erweitert. Insbesondere wurde versucht die flächenhafte Verteilung der Schneedecke besser zu erfassen, um eine Validierung der verwendeten Schnee- und Eisschmelzmodelle zu ermöglichen. Die Erfassung der Schneetiefe im Hochgebirge stellt eine hohe messtechnische Herausforderung dar. Während auf einem Gletscher die Messung der Schneetiefe mit einer Sonde recht gut bestimmt werden kann, stellt die Messung außerhalb der Gletscherflächen eine nicht leicht durchführbare Aufgabe dar. Der oft sehr unterschiedliche Untergrund (grobes Blockwerk, steile Hänge etc.) lässt eine eindeutige Bestimmung der Schneetiefe nicht zu.

Für das Jahr 2005 wurde trotzdem versucht die räumliche Verteilung der Schneetiefe im gesamten Einzugsgebiet des $\mathrm{Pe}$ gels Goldbegkees während des Schneedeckenabbaues durch regelmäßige Sondierung zu er-

Tab. 2: Horizontale und vertikale Eisbewegung am Goldbergkees und Kleinfleißkees im Haushaltsjahr 2005/06

\begin{tabular}{|c|c|c|c|c|c|c|c|}
\hline Pegel & $\begin{array}{c}\text { Hang- } \\
\text { neigung } \\
{\left[^{\circ}\right]}\end{array}$ & $\begin{array}{c}\text { x-Richtung } \\
\text { [m] }\end{array}$ & $\begin{array}{c}\text { y-Richtung } \\
\text { [m] }\end{array}$ & $\begin{array}{c}\text { Änderung } \\
\text { Horizontal } \\
\text { [m] }\end{array}$ & $\begin{array}{c}\text { Änderung } \\
\text { Vertikal } \\
\text { [m] }\end{array}$ & $\begin{array}{c}\text { Ablation } \\
{[\mathrm{m}]}\end{array}$ & $\begin{array}{c}\text { Vertikal- } \\
\text { bewegung } \\
\text { [cm] }\end{array}$ \\
\hline $\mathrm{S} 11$ & 24 & 0.5 & 0.2 & $0.5 \pm 0.01$ & $-0.7 \pm 0.10$ & -0.8 & 12 \\
\hline F03/3 & 10 & 2.3 & 0.4 & $2.3 \pm 0.01$ & $-0.6 \pm 0.16$ & -0.9 & 27 \\
\hline F03/2 & 12 & 2.1 & 0.4 & $2.1 \pm 0.01$ & $-0.8 \pm 0.15$ & -1.3 & 57 \\
\hline F04/3 & 8 & 3.7 & 0.5 & $3.7 \pm 0.04$ & $-0.9 \pm 0.24$ & -1.6 & 74 \\
\hline F04/2 & 13 & 5.2 & 1.2 & $5.2 \pm 0.01$ & $-0.3 \pm 0.26$ & -1.2 & 81 \\
\hline F04/4 & 8 & 4.8 & 0.6 & $4.8 \pm 0.01$ & $-0.7 \pm 0.24$ & -0.8 & 11 \\
\hline $99 / 7$ & 5 & 4.0 & 0.4 & $4.0 \pm 0.01$ & $-1.1 \pm 0.22$ & -0.3 & -77 \\
\hline F04/1 & 11 & 5.9 & 1.2 & $5.9 \pm 0.01$ & $-0.7 \pm 0.29$ & -1.6 & 97 \\
\hline F03/1 & 12 & 3.3 & 0.7 & $3.3 \pm 0.01$ & $-0.9 \pm 0.20$ & -1.7 & 74 \\
\hline G03/2 & 18 & 2.4 & 0.8 & $2.4 \pm 0.01$ & $-1.2 \pm 0.16$ & -1.4 & 14 \\
\hline G05/6 & 8 & 0.1 & 0.0 & $0.1 \pm 0.01$ & $-1.8 \pm 0.08$ & -1.9 & 14 \\
\hline G03/7 & 11 & 0.2 & 0.0 & $0.2 \pm 0.01$ & $-1.3 \pm 0.09$ & -1.4 & 10 \\
\hline G04/4 & 9 & 1.4 & 0.2 & $1.4 \pm 0.01$ & $-1.1 \pm 0.13$ & -1.3 & 11 \\
\hline G04/3 & 5 & 1.3 & 0.1 & $1.3 \pm 0.01$ & $-1.0 \pm 0.12$ & -0.8 & -20 \\
\hline G03/4 & 3 & 0.3 & 0.0 & $0.3 \pm 0.02$ & $-1.6 \pm 0.10$ & -1.8 & 20 \\
\hline $\mathrm{G} 04 / 2$ & 13 & 1.1 & 0.3 & $1.1 \pm 0.02$ & $-1.2 \pm 0.12$ & -1.6 & 38 \\
\hline G03/11 & 14 & 1.8 & 0.4 & $1.8 \pm 0.01$ & $-1.0 \pm 0.14$ & -1.8 & 77 \\
\hline G04/5 & 15 & 1.7 & 0.4 & $1.7 \pm 0.03$ & $-1.9 \pm 0.16$ & -1.4 & -46 \\
\hline
\end{tabular}




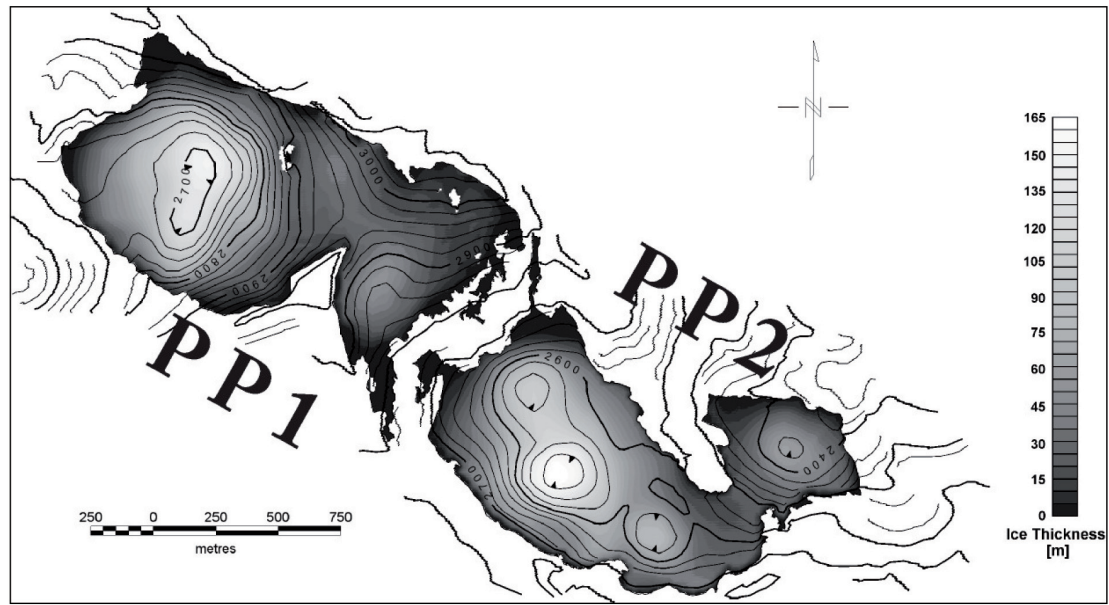

Abb. 6: Eismächtigkeit von Goldbergkees und Kleinfleißkees im Jahr 2003. Die Messungen wurden mit einem Bodenradar durchgeführt (Binder et al., 2009)
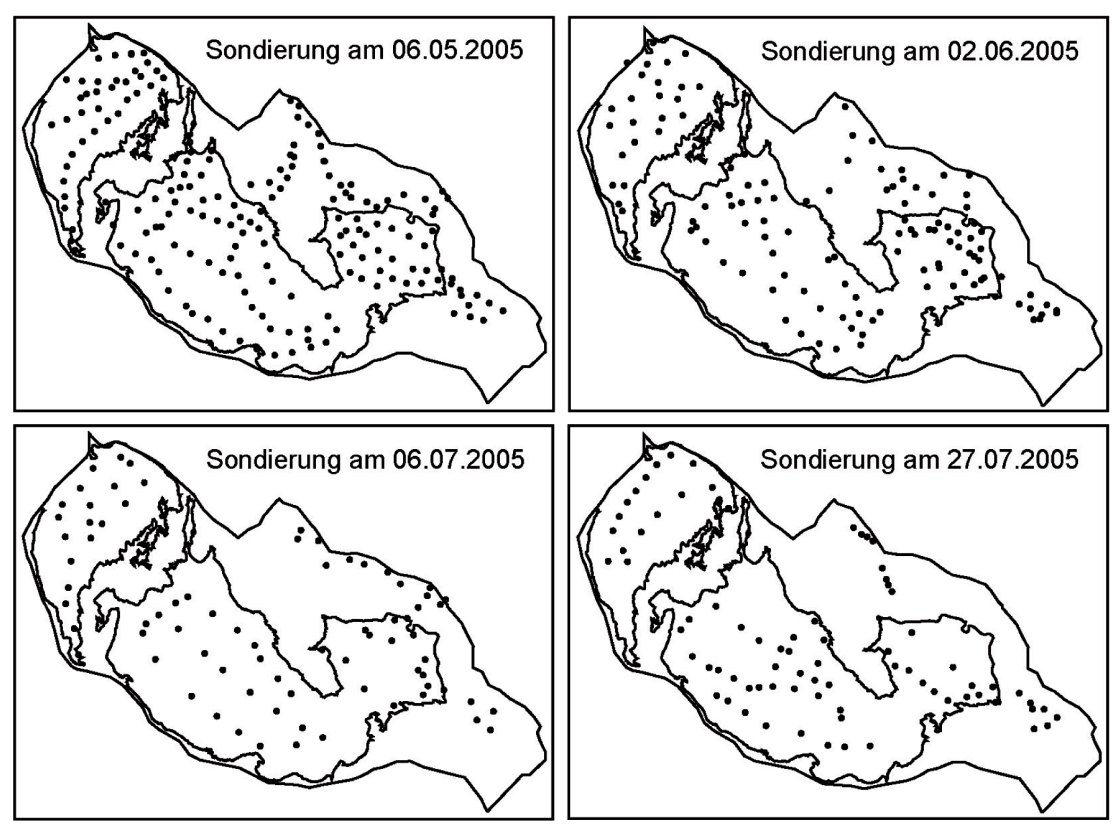

Abb. 7: Schneetiefensondierung im Bereich des Einzugsgebietes Pegel Goldbergkees im Frühjahr 2005

fassen. Die Sondierungen konnten am 6.5., 2.6., 6.7. und am 27.7.2005 durchgeführt werden. Das Messnetz ist aus Abbildung $7 \mathrm{zu}$ ersehen. Im Vergleich zum Gletscher selber ist die Punktdichte außerhalb wesentlich geringer. Das ist vorwiegend durch die Unwegsamkeit des Geländes zu erklären. Trotzdem konnte eine sehr gute Datengrundlage für die Validierung der Modelle gewonnen werden (siehe Koboltschnig et al., 2008).

\section{Charakterisierung des Zusammenhangs Klima - Gletscher im Bereich des Sonnblicks}

Der Zusammenhang Klima-Gletscher wurde bereits vielfach untersucht. Mehrere Untersuchungen in den Alpen haben gezeigt, dass die Massenbilanz der Gletscher derzeit vorwiegend von der Sommerbilanz bestimmt wird (z. B. Schöner et al., 2000, Kuhn 2004, Hermann und Kuhn, 1990). Es ist jedoch zu beachten, dass dieser Zusammenhang nicht als zeitlich konstant zu betrachten ist (Schöner et al., 2001). Die Sommerbilanz eines Gletschers wird im wesentlichen durch die Energiebilanz an der Gletscheroberfläche beschrieben. Um die Bedeutung der einzelnen Komponenten der Energiebilanz abschätzen zu können wurden mehrmals Messungen der Energiebilanz über Gletscher durchgeführt. In Tabelle 3 wird eine Übersicht über mehrere Messungen gezeigt, in der auch Messungen vom Goldbergkees im Jahr 2003 (Hynek, 2007) enthalten sind. Eindeutig wird durch alle Messungen die hohe Bedeutung der Strahlungsbilanz (wobei hier fast ausschließlich die kurzwellige Strahlungsbilanz zum tragen kommt) belegt die zu zirka 70 - $90 \%$ die Energiebilanz, und damit die sommerliche Ablation, bestimmt.

Eine interessante Fragestellung betrifft die Klimasensitivität der Gletscher, insbesondere die Temperatursensitivität. Die Temperatursensitivität bezeichnet die Änderung der Gletschermassenbilanz bei einer Änderung der Lufttemperatur. Für die Gletscher der Alpen wurde das von Oerlemans (z.B. Oerlemans et al., 1998) eingehend mittels eines physikalisch basierten Massenbilanzmodells untersucht. Ein vereinfachter Ansatz kann mittels eines statistischen Modells verfolgt werden. Abbildung 8 zeigt diesen Zusammenhang für die mittlere Sommertemperatur und die Sommermassenbilanz für verschiedene Gletscher in Österreich an Hand eines einfachen linearen Regressionsmodells. Die Gegenüberstellung für die 4 Gletscher Jamtalferner (Silvretta), Vernagtferner (Ötztaler Alpen), Wurtenkees (Hohe Tauern) und Goldbergkees (Hohe Tauern) führt zu dem Schluss, dass das Goldbergkees die höchste Temperatursensitivität besitzt, der Vernagtferner die geringste. Dieses Ergebnis stimmt mit den Erwartungen überein, da für Glet- 
Tab. 3: Vergleich der Ergebnisse von Energiebilanzmessungen an verschiedenen alpinen Gletschern (RAD = Strahlungsbilanz, SH = Fühlbarer Wärmestrom, LH = Latenter Wärmestrom, RAIN = Wärmeeintrag durch Regen, Melt calc = berechnete Schmelze, alle Angaben sind in \%); aus Hynek (2007)

\begin{tabular}{|c|c|c|c|c|c|c|c|}
\hline Author & Tanzer & $\begin{array}{c}\text { Greuell, } \\
\text { Oerle }\end{array}$ & $\begin{array}{c}\text { Hoinkes, } \\
\text { Unt }\end{array}$ & Oerlemans & Greuel & Fitzgerald & Hynek \\
\hline $\begin{array}{l}\text { Glacier } \\
\text { Elevation } \\
\text { Method }\end{array}$ & $\begin{array}{l}\text { Hintereis } \\
2960 \\
\text { Gradient }\end{array}$ & $\begin{array}{l}\text { Hintereis } \\
2500 \\
\text { Gradient }\end{array}$ & $\begin{array}{c}\text { Vernagt } \\
2973 \\
\text { Gradient }^{\star}\end{array}$ & $\begin{array}{c}\text { Morteratschg. } \\
2600 \\
\text { Bulk }\end{array}$ & $\begin{array}{l}\text { Pasterze } \\
2400 \\
\text { Bulk }\end{array}$ & $\begin{array}{l}\text { Autumngl } \\
3200 \\
\text { Bulk }\end{array}$ & $\begin{array}{c}\text { Goldberg } \\
2500 \\
\text { Bulk }\end{array}$ \\
\hline RAD & 69 & 91 & 81 & 85 & 78 & 75 & 67 \\
\hline $\mathrm{SH}$ & 33 & 11 & 18 & 20 & 23 & 25 & 34 \\
\hline LH & -3 & -2 & 1 & -5 & 4 & -2 & -1 \\
\hline RAIN & 1 & 0 & 0 & 0 & -5 & 2 & 0 \\
\hline Melt calc & 100 & 100 & 100 & 100 & 100 & 100 & 100 \\
\hline
\end{tabular}
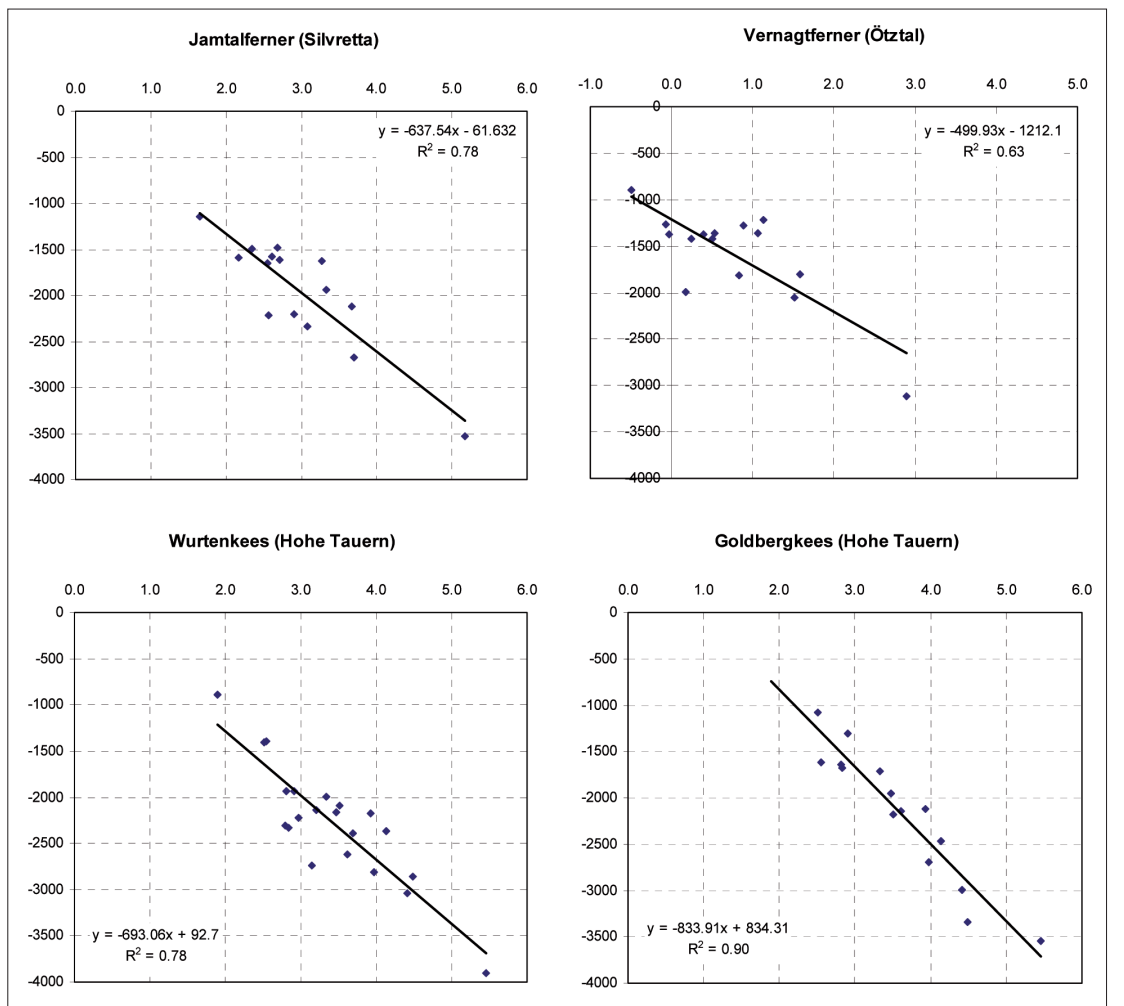

Abb. 8: Beziehung zwischen spezifischer Sommerbilanz und mittlerer Sommertemperatur (Mai bis September) für verschiedene Gletscher in Österreich für die gemeinsame Periode 1989-2003 (Temperaturwerte von HISTALP Datensatz, siehe Auer et al., 2007, Schöner und Böhm 2007)

scher in niederschlagsreicheren Regionen im Allgemeinen eine höhere Temperatursensitivität zu erwarten ist (Oerlemans et al., 1998). Nimmt also die Lufttemperatur um $1^{\circ} \mathrm{C}$ zu so ändert sich die spezifische Sommermassenbilanz des Vernagtferners um $-500 \mathrm{~mm}$ während sie sich am Goldbergkees um - 835 mm ändert. Interessant an diesem Vergleich der 4 Gletscher ist auch der deutliche Unterschied zwischen den benachbarten Gletschern Wurtenkees und Goldbergkees. Auch das Wurtenkees hat auf Grund seiner Lage in einem vergleichsweise (zum Goldbergkees) niederschlagsärmeren Gebiet eine geringere Temperatursensitivität als das Goldbergkees. Bei dieser Überlegung ist aber zu beachten, dass die Temperatursensitivität nicht nur den fühlbaren Wärmestrom beschreibt sondern die Energiebilanz insgesamt. Dieser Zusammenhang wurde mehrmals beschrieben (z. B. Ohmura 2001) und konnte auch für das Goldbergkees durch die Messungen von Hynek (2007) bestätigt werden.

\section{Schlussfolgerungen}

Die glaziologischen Messungen im Bereich des Rauriser Sonnblicks (Hohe Tauern) stellen gemeinsam mit den Messungen des Sonnblick Observatoriums ein klimatologisch und hydrologisch sehr detailliertes Monitoring in den Alpen dar. Der Alpenhauptkamm als markante Klimascheide verursacht eine sehr hohe räumliche Variabilität des Gletscherverhaltens, so dass die Messung an mehreren Gletschern über den Alpenhauptkamm hinweg sinnvoll ist. Die Massenbilanz der Gletscher ist sehr stark durch die Orographie beeinflusst und spiegelt die klimatologische Varabilität in dieser Region deutlich wider. Da sich die Gletscher im Bereich des Sonnblicks in einer, für die Alpen, sehr niederschlagsreichen Region befinden, zeigen sie eine deutlich erhöhte Klimasensitivität im Vergleich zu anderen Gletscher in Österreich. Eismächtigkeitsmessungen zeigen für die kleinen Gletscher des Sonnblickgebietes noch sehr große Eistiefen (etwas 150m Maximaltiefe für das $1.5 \mathrm{~km}^{2}$ große 
Goldbergkess. Die sehr gute klimatologische/hydrologische/glaziologische Datenlage im Sonnblickgebiet, macht es sehr gut für die Kalbrierung und Validierung von Schnee- und Eisschmelzmodellen geeignet.

\section{Danksagung}

Das Gletschermonitoring im Sonnblickgebiet wird im Auftrag des Lebensministeriums durchgeführt (Projekt MOMBASA, Zahl:54 4768/1-V/4/04) und wird durch Mittel der Eigenforschung der Zentralanstalt für Meteorologie unterstützt. Im Rahmen des Projektes
SNOWTRANS fand das Monitoring auch Unterstützung durch die Österreichische Akademie der Wissenschaften im Rahmen der Hydrologie Österreichs (HÖ29).

Zentralanstalt für Meteorologie und Geodynamik, Klimaabteilung, Wien, Österreich

${ }^{2}$ Technische Universität Wien, Institut für Geodäsie und Geophysik, Wien, Osterreich

3 Universität für Bodenkultur, Department für Wasser, Atmosphäre und Umwelt, Wien, Österreich ${ }^{4}$ Internationale Forschungsgesellschaft INTERPRAEVENT, Klagenfurt, Osterreich

\section{Korrespondenz:}

Wolfgang Schöner

Zentralanstalt für Meteorologie und

Geodynamik, Klimaabteilung, Wien

E-Mail: wolfgang.schoener@zamg.ac.at

\section{LITERATUR}

Auer I, Böhm R, Leymüller M, Schöner W (2002) Auer I, Böhm R, Leymúler M, Schoner W (2002)

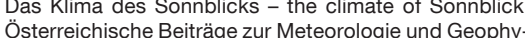
Österreichische
sik, Heft 29

sik, Heft 29 Auer I, Böhm R, Jurkovic A, Lipa W, Orlik A Auer I, Böhm R, Jurkovic A, Lipa W, Orlik A
Potzmann R, Schöner W, Ungersböck M, Matulla C Briffa K, Jones PD, Efthymiadis D, Brunetti M, Nann T, Maugeri M, Mercalli L, Mestre O, Moisselin J-M Begert M, Müller-Westermeier G, Kveton V, Bochnicek O, Stastny P, Lapin M, Szalai S, Szentimrey T, Cegnar T, Dolinar M, Gajic-Capka M, Zaninovic K, Majstorovic Z, Nieplova E (2007) HISTALP - Historica instrumental climatological surface time series of the greater Alpine region 1760-2003. International Journa of Climatology, 27: 17-46.

Binder D (2008) Bestimmung der Eisdickenverteilung sowie des Gesamteisvolumens dreier Gletscher in der Goldberggruppe auf der Basis von Ground Penetrating Radar Daten, Diplomarbeit, Universität Wien.

Binder D, Brückl B, Roch KH, Behm M, Schöner $W$ and Hynek B (2009, accepted) Determination of Total Ice Volume and Ice Thickness Distribution of Two tal Ice Volume and Ice Thickness Distribution of Two Glaciers in the Hohe Tauern region (Eastern Alps) from Ground Penet

Brückl E und Bittmann $O$ (1977) Die Ergebnisse der seismischen Gletschermessungen im Bereich der der seismischen Gletschermessungen im Bereich de Goldbergguppe (Hohe Tauen) in den Jahren 1971 und 1972. Arbeiten aus der Zentralanstalt für Meteorologie und Geodynamik, Heft 25, Wien

Dirmhirn I und Sauberer F (1952) Der Strahlungshaushalt horizontaler Gletscherflächen auf dem Hohen Sonnblick. Geogr. Ann. 34, 261-290.

Dirmhirn I (1961) Ergebnisse von Albedountersuchungen im Sonnblickgebiet. Wetter und Leben, Sonderheft 9, 115-119

Dyurgerov M and Meier MF (2005) Glaciers and the Changing EarthSystem: A 2004 Snapshot. Occasiona Paper 58, Institute of Arctic and Alpine Research, University of Colorado, Boulder, 118pp.

Escher Vetter H, Braun LN, Siebers M and Weber M (2005) Water balance of the Vernagtferner high alpine basin based on long-term measurements and modelling. Landschafsökologie und Umweltforschung, TU-Braunschweig, Vol 48, 19-32.

Finsterwalder R (1953) Die zahlenmäßige Erfassung des Gletscherrückganges an Ostalpengletschern. Zeit- schrift für Gletscherkunde und Glazialgeologie, II, Heft

Gattermayr W, Kuhn M, Weilguni V (2005) Schneemessstellen und beobachtete Gletscher. Hydrologischer Atlas Österreichs, Wien

Haeberli W, Hoelzle M, Paul F and Zemp M (2007) Integrated monitoring of mountain glaciers as key indicators of global climate change: the European Alps. Annals of glaciology 46, 150-160.

Hermann A und Kuhn M (1990) Schnee und Eis. In: Baumgartner A. und H.J. Liebscher (Hrsg.). Lehrbuch der Allgemeinen Hydrologie. Bornträger, Berlin.

Hoinkes H (1970) Methoden und Möglichkeiten von Massenhaushaltsstudien auf Gletschern - Ergebniss der Messreihe Hintereisferner 1953-1968. Zeitschrift für Gletscherkunde und Glazialgeologie, Band VI, 37-90.

Holmlund P, Jansson P und Pettersson R (2005) A re-analysis of the 58 year mass balance record of Storglaciären, Sweden. Ann. Glaciol. 42: 389-394.

Hübl J (1912) Die stereophotogrammetrische Aufnahme des Goldberggletschers im August 1909. Denkmatische Classe, 87. Band, Wien, 153-160.

Classe, 87. Band, Wien, 153-160. Hynek B (2007) Messungen zum Energiehau
am Goldbergkees. Diplomarbeit, Universität Wien.

IPCC (2007) Climate Change 2007: The Physical Science Basis. Contribution of Working Group I to the Fourth Assessment Report of the Intergovernmental Panth Assessment Report of the Intergovernmental Panel on Climate Change [Solomon, S., D. Qin, M. Manning, Z. Chen, M. Marquis, K.B. Averyt,M. Tignor
and H.L. Miller (eds.)]. Cambridge University Press, Cambridge, United Kingdom and New York, NY, USA, $996 \mathrm{pp}$

Kaser G, Fountain A, Jansson P (2003) A manua for monitoring the mass balance of mountain glaciers with particular attention to low latitude characteristics. A contribution form the International Commission on Snow and Ice (ICSI) to the UNESCO-HKH-Friend program. UNESCO IHP-VI, Technical Document 59.

Koboltschnig GR, Schoener W, Zappa M, Kroisleitner C, Holzmann $\mathrm{H}$ (2008) Runoff modelling of the glacierized alpine Upper Salzach basin (Austria): Multicriteria result validation. Hydrological Processes 22: 3950-3964. DOI: 10.1002/hyp.7112

Kuhn M (2004) Die Reaktion der österreichischen Kuscer und inres Abflusses auf Änderungen vo Temperatur und Niederschlag. Österreichische Wasser- und Abfallwirtschaft 56, Heft 1-2, 1-7. Lichtenecker N (1936) a: Gletscheruntersuchungen in der Sonnblickgruppe 1930 bis 1934 . Anz.Akad.Wiss. Abt.2, 73, 96-98.

Lichtenecker N (1936) b: Neuere Gletscherstudien in der Sonnblickgruppe. 44. Jahresbericht des Sonnblickvereins für das Jahr 1935, 13-37.

Lichtenecker N (1938) Messungen am Goldbergkees (Sonnblickgruppe) in den Sommern 1936 und 1937. 46.Jahresber. des Sonnblick Vereines 1937, 16 22.

Oerlemans und 10 andere. (1998) Modelling the response of glaciers to climate warming. Clymate Dynamics 14(4), 267-274.

Ohmura A (2001) The physical basis for the temperature based melt-index method. Journal of Applied Meteorology 40/4, 753-761

Penck A (1897) Gletscherstudien im Sonnblickgebiete.

Sauberer F (1939) Strahlungsumsatz- und Albe-

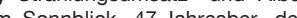
Sonnblick Vereins, 1938 , 9-14.

Sauberer F und Dirmhirn I (1950) Die Bedeutung des Strahlungsfaktors für den Gletscherhaushalt. Wetter und Leben, Jahrgang 2, Heft 11-12, 248-261.

Schöner W, Auer I and Böhm R (2000) Climate variability and glacier reaction in the Austrian eastern Alps. Annals of Glaciology, Vol. 31, 31-38.

Schöner W, Böhm R (2007) A statistical massbalance model for reconstruction of LIA ice mass for glaciers in the European Alps. Annals of Glaciology, Vol. 46, 161-169.

Schöner W, Auer I, Böhm R (2009, accepted) Long term trend of snow depth at Sonnblick (Austrian Alps) and its relation to climate change. Hydrological Processes. Special Issue: Mountain Hydrology and Snow Seasonality.

Tollner H (1978) Die sommerliche Klimaverwerfung 1950 und ihre Folgen auf das Wasserdargebot des vergletscherten Hochgebirges. Arb. ZAMG 32, ITAMRauris 1976, 93: 1-6.

Zemp M (2006) Glaciers and climate - spatio-temporal analysis of glacier fluctuations in the European Alps after 1850 . Schriftenreihe Physische Geographie Alaziale 